\title{
Engineering Approach to Calculating QoS of Server with Self-Similar Incoming Traffic Based on Recursive Scalable Poisson Model
}

\author{
Vladimir Lokhmotko', Sabina Rudinskaya ${ }^{2}$ \\ ${ }^{1}$ Federal Communications Agency (Rossvyaz), The Bonch-Bruevich Saint-Petersburg State University of Telecommunications, \\ Saint-Petersburg, Russian Federation \\ ${ }^{2}$ Department of Education, Belarusian State Academy of Telecommunications, Minsk, The Republic of Belarus
}

\section{Email address:}

1vv1950@tut.by (V. Lokhmotko), sabina.rudin@mail.ru (S. Rudinskaya)

\section{To cite this article:}

Vladimir Lokhmotko, Sabina Rudinskaya. Engineering Approach to Calculating QoS of Server with Self-Similar Incoming Traffic Based on Recursive Scalable Poisson Model. American Journal of Networks and Communications. Vol. 6, No. 6, 2017, pp. 79-86.

doi: 10.11648/j.ajnc.20170606.11

Received: November 21, 2017; Accepted: November 27, 2017; Published: January 2, 2018

\begin{abstract}
To date the prospects for using the accumulated over many years mathematical and software for the modeling of telecommunications with the Poisson input flow are under a big question. The matter is that a new fractal queuing theory is already on the threshhold. This article formulates and solves the problem of application of a queuing system model with a Poisson incoming flow for the purposes of server modeling described by QS with self-similar incoming traffic of the "fractal Brownian motion" type (according to Norros). Based on the results of the morphological analysis, the Norros model was decomposed into Poisson components connected by a scalable recurrence scheme. The variance of the number of packets in the server, raised to the power determined by the Hurst parameter acts as the similarity coefficient of fractal and Poisson QSs. The method for rescaling Poisson solutions into fractal solutions was constructed on the basis of the similarity coefficient. According to this method in order to find the fractal delay of access, the Poisson delay should be multiplied by the similarity coefficient, and to estimate the probability of packet loss, it is necessary to extract a root of degree equal to the similarity coefficient from classical exponential losses. The scope of the re-scaling method focuses on the pre-project stages of creating telecommunications, where there is no need for high accuracy of simulation results.
\end{abstract}

Keywords: Self-Similarity, Norros Model, Hurst Parameter, Similarity Coefficient, Recurrence Model, Two-Parameter Exponential Distribution, Access Delay, Loss Probability

\section{Introduction}

Despite the immense popularity of researching the selfsimilar properties of packet traffic and the duration (more than two decades) of its study, we have to admit that many questions and tasks are not yet detailed to the level of applied engineering $[1,2]$. This was the reason for the shortage of common universally accepted methods for modeling, calculating and predicting QoS of self-similar traffic, simple enough, accurate and convenient to use. In particular, for the preparation of business plans and investment projects of infocommunications on an IP platform, express methods and compact analytical models for servicing self-similar traffic are needed.
Many modeling tools (MT) of infocommunications, which were initially oriented to the Poisson incoming flow, are of little use in the case of heavy traffic. Therefore, the task of renovating the Poisson-oriented mathematical and software MT, developed for many years under the conditions of nonPoisson, in a particular case self-similar traffic, remains topical.

In this paper, we formulate the problem of restructuring queueing system with self-similar traffic (in the treatment of Norros [3]) for separation of transparent components with a Poisson incoming flow [4].

The relevance of the issues covered in this article from this theoretical point of view is dictated by a large or infinite variance of self-similar random processes, but with a 
practical need for simple analytical methods for estimating QoS indicators of infocommunications on an IP platform in a self-similar traffic environment.

\section{Problem Definition}

The known formula for the average waiting time of the packet in the queue to the server simulated by the $f B / G / 1 / \infty$ QS for an arbitrary $(G)$ law for the distribution of the servicing time of the self-similar incoming $(f B)$ traffic of the "Fractal Brownian Motion" (FBM) [3, 5] is

$$
W=\frac{h}{d} \cdot \frac{\rho^{a}}{(1-\rho)^{b}}, a=\frac{1}{2(1-H)}, \quad b=\frac{H}{1-H},
$$

where: $H$ - Hurst index, which characterizes the degree of self-similarity of the incoming flow;

$d=2 /\left(1+C_{b}^{2}\right)$ - factor, determined by the coefficient $C_{b}$ of variation of service time;

$\rho=\lambda V / C=\lambda h-$ server utilization factor, as a function of the $\lambda$ intensity of the incoming flow, the average packet processing time $h$ (transmission), average packet volume $V$ and bit rate $C, \rho<1$.

In addition to $(0,5<H<1)$ self-similar traffic, formula (1) for $H=0,5$ generalizes the well-known Poisson models of mean waiting time in the buffer [4], corresponding to:

- for $C_{b}=0$ - QS $M / D / 1 / \infty$ with deterministic service;

- for $C_{b}=1-\mathrm{QS} M / M / 1 / \infty$ with exponential service ;

- for an arbitrary $C_{b}-\mathrm{QS} M / G / 1 / \infty$ type.

Required:

- to transform the packet delay formula (1) into terms and notations of classical QS with a Poisson $(M)$ incoming stream;

- to construct a similar Poisson approximation for calculating the probability of loss of a fractal-type packet.

\section{Related Work}

Numerous approaches to the estimation of QoS indicators of a network of self-similar traffic are known [1,6]. The authors of this article adhere to the approach $[5,7]$, based on the preservation of earlier investments in queuing theory by adapting traditional modeling methods to the conditions of self-similar traffic. By making minor modifications, it becomes possible to reuse the methods and tools of telecommunication modeling that were developed in the past and with which the experience has been accumulated.

Innovative ideas for solving the problem of high-quality servicing of "heavy" traffic should be expected from the fractal queuing theory, [1, 2]. However, the developed analytical fractal models (statistical and integro-differential) are still far from the engineering level of detailing, and the corresponding simulation models still do not allow solving the problem of slow convergence of estimates of random variables with a large variance.

In the problems of modeling the decreasing complexity, no special problems are visible, because generalized distribution functions of random variables (for example, gamma distribution, Erlang distribution, Weibull distribution and others [8]), if necessary, are easily transformed into a simpler, classical exponential distribution. The situation is more complicated with the solution of inverse problems, when it is necessary to simulate the process of servicing self-similar traffic using Poisson's tools.

The prototype of the approach [5, 7] can be the method [9] of equivalent replacements used by telephone engineers for an approximate transition from queuing systems with waiting, systems with repeated calls or systems with bypass directions and overload to a simple, well-studied the 1-st Erlang formula.

A similar method for transforming QSs with self-similar traffic into QS with fractal traffic can be constructed on the principle of similarity of systems [10], which assumes the reduction of the initial problem of unorganized complexity to the problem of organized simplicity. For practice, this is identified with the integration of the new MT from the existing set of simpler, well-known and well-developed software subsystems with the possibility of subsequent rescaling of the resulting solution under the initial conditions of the fractal modeling problem.

As the operational model, Little's formula [4] is used in this paper to link streaming, capacitive and time indicators and is a paradigm of the family of models that is valid for any QS, any nature of the flow of applications, any distribution of service time and any service discipline. A key element of the Little's model is the Norros model [3] for estimating the average queue length.

\section{Recurrent Delay Model}

\subsection{Features of the Chosen Approach}

A fractal (self-similar) process is usually understood as a random process whose statistical characteristics manifest the properties of scaling, long-term dependence, and slowly decreasing dispersion [2].

The specifics of fractal structures $[1,2]$ are known:

- invariance with respect to the change in scale and size, which is an inherent property of self-similarity;

- fractional, not integer dimension;

- the presence of the word fractal in addition to the value "fractal" ("fractional"), as well as the values "recursive";

- the possibility of mathematical formalization of scale invariance (scaling) by power laws with fractional exponents, which are known as "generators" of self-similarity;

- presence of fragments, like an entire figure - in the structural plan.

Practical implementation of the chosen approach will consist in the composition of functions of new QoS-models from the available set of simpler Poisson models, and the simulation technology is reduced to the rescaling of Poisson solutions. 


\subsection{Integer Case}

Let us analyze the formula for the average length of the queue, which, according to Little

$$
L=\lambda \cdot W
$$

We introduce the auxiliary index series

$$
1,2,3, \ldots, n, \ldots
$$

and for each index of the sequence (3), proceeding from the formula (1) (for the sake of simplicity we choose the exponential service, $d=1$ ), by induction we calculate Table 1 .

\begin{tabular}{|c|c|c|c|c|}
\hline \multirow{2}{*}{ Index $n$} & \multicolumn{2}{|l|}{ Exponents } & \multirow{2}{*}{ Hurst parameter $\boldsymbol{H}$} & \multirow{2}{*}{ Average queue length } \\
\hline & $a$ & $b$ & & \\
\hline 1 & 2 & 1 & 0,5 & $L_{1}=\rho^{2} /(1-\rho)$ \\
\hline 2 & 3 & 3 & 0,75 & $L_{2}=\rho^{3} /(1-\rho)^{3}$ \\
\hline 3 & 4 & 5 & $0,833 .$. & $L_{3}=\rho^{4} /(1-\rho)^{5}$ \\
\hline$\cdots$ & $\cdots$ & $\ldots$ & $\ldots$ & $\ldots$ \\
\hline$n$ & $n+1$ & $2 n-1$ & $1-0,5 / n$ & $L_{n}=\rho^{n+1 /(1-\rho)^{2 n-1}}$ \\
\hline
\end{tabular}

Table 1. Morphological analysis of the function (1) for integer values of exponents $a$ and $b$.

Table 1 shows that the function (1) is endowed with a recurrent structure with the following properties:

- for the selected (in the 4th column) values of the Hurst parameter, the average queue lengths $L_{1}, L_{2}, \ldots . L_{n}$, (in the 5 th column) form a geometric progression, i.e. a numerical sequence, each of whose numbers is equal to the previous one, multiplied by a constant for a given progression number $q$, which is the denominator of the geometric progression,

$$
L_{n}=L_{1} \cdot q^{n-1}
$$

- the first member of the progression

$$
L_{1}=\rho^{2} /(1-\rho)
$$

has a simple physical meaning, namely the average queue length in the QS buffer $M / M / 1 / \infty$ [4];

- denominator of progression

$$
q=D=\frac{\rho}{(1-\rho)^{2}}
$$

is the variance $D[4]$ of the number of packets in the QS $M / M / 1 / \infty$;

- denominator of the progression to the power of $(n-1)$

$$
q^{n-1}=m=L_{f B / M / 1}: L_{M / M / 1}=L_{n}: L_{1}
$$

is a similarity coefficient $m$, i.e. multiplicative factor, which is the ratio of the average queue lengths of the fractal QS $f B / M / 1 / \infty$ and the Poisson QS $M / M / 1 / \infty$;

- the square of the $n$-th member of the geometric sequence is equal to the product of the adjacent $(n-1)$-th and $(n+1)$-th members.

Proceeding from formulas (2) and (4), the average delay (1) of access to the server is represented as the $n$-th (common) geometric progression member

$$
W_{2}=L_{2} / \lambda=h \cdot L_{2} / \rho=1 \cdot 27 / 0,75=36 \mathrm{~ms}(\mathrm{QS} f B / M / 1 / \infty),
$$

Engineering interpretation of calculation results:

$$
W_{n}=W_{1} \cdot q^{n-1}, n=\frac{1}{2(1-H)}=1,2,3 \cdots
$$

the first member of which $W_{1}=h \cdot \rho /(1-\rho)$ corresponds to the average waiting time of the packet in the QS buffer $M / M / 1 / \infty$.

The index $n$ is derived from the Hurst parameter and is defined as the nearest integer from (8).

Comparing the original fractional-power function (1) with its recurrence analog (8), we can verify that they are completely identical for integer values $n=1,2,3 \ldots$

Example 1. Given: server load $\rho=0.75$; exponential ( $d=$ 1) maintenance; average packet processing time $h=1 \mathrm{~ms}$; self-similar incoming traffic with Hurst parameter $H=0.75$. It is required to evaluate QoS-indicators using the recurrence model described above.

Consistently is determined the following:

1) serial number $n$ (row index) $n=\frac{1}{2(1-H)}=\frac{1}{2(1-0,75)}=2$;

2) the first member of the progression $L_{1}=\rho^{2} /(1-\rho)=0,75 \cdot 0,75:(1-0,75)=2,25$;

3) denominator of progression $q=\frac{\rho}{(1-\rho)^{2}}=\frac{0,75}{(1-0,75)(1-0,75)}=12$;

4) similarity factor $m=q^{n-1}=12^{2-1}=12$;

5) average queue length $L_{2}=L_{1} \cdot q^{n-1}=2,25 \cdot 12^{2-1}=27$.

In conclusion, according to Little's formulas [4], there are:

6) the average number of packets in the systems:

$$
\begin{gathered}
\bar{N}_{2}=\rho+L_{2}=0,75+27=27,75(\mathrm{QS} f B / M / 1 / \infty), \\
\bar{N}_{1}=\rho+L_{1}=0,75+2,25=3(\mathrm{QS} M / M / 1 / \infty),
\end{gathered}
$$

7) average access delay - the first member $L_{1}=2,25$ of the progression corresponds 
to the average length of the QS queue $M / M / 1 / \infty$ with a Poisson incoming flow, for which $H=0.5$;

- the denominator $q$ of the progression mathematically corresponds to the growth rate of the progression, and physically the variance $D=q=12$ of the number of packets in the QS $M / M / 1 / \infty$ with the Poisson incoming flow;

- the average queue length $L_{1}$ of the QS queue with the Poisson incoming flow (QS $M / M / 1 / \infty, \mathrm{H}=0.5$ ) is slightly larger than 2 packets, and the standard deviation of the number of packets in the system $\sigma=\sqrt{D}=\sqrt{12} \approx 3,46$;

- for the accepted conditions, the average queue length of the QS queue with the self-similar $(H=0.75)$ incoming flow exceeds that for Poisson traffic $(H=0.5)$ by more than an order of magnitude;

- since the similarity factor is $m=12$, then for an equivalent replacement of the QS buffer $f B / M / 1 / \infty$ (with self-similar incoming traffic), 12 QS buffers $M / M / 1 / \infty$ with Poisson traffic are required;

- by capacity (the number of packets being processed + waiting), the QS $f B / M / 1 / \infty$ exceeds the QS $M / M / 1 / \infty$ by almost an order of magnitude $\left(\bar{N}_{2} / \bar{N}_{1}=27,75: 3=9,25\right)$;

- other things being equal, self-similar traffic proves to be more resource-intensive and less qualitatively served in comparison with Poisson traffic. Therefore, to maintain the quality of service of self-similar incoming traffic corresponding to Poisson, it is required to increase the server's performance by more than an order of magnitude. Thus, the fractal mean delay of access to the server can be represented as the product of Poisson components:

$$
W=W_{2}=h \cdot \bar{N}_{1} \cdot D^{n-1},
$$

the average number of packets in the system by the variance of the number of packets with the additional presence of the average processing time $h$ and the Hurst parameter $H$.

\subsection{The Similarity Coefficient}

As follows from (7), the similarity coefficient of fractal (1) and recurrent (8) or (9) models:

- is mathematically represented as a power function of the utilization factor and the Hurst parameter $H$

$$
m=D^{n-1}=\left[\frac{\rho}{(1-\rho)^{2}}\right]^{n-1}, n=\frac{1}{2(1-H)}, 0,5 \leq H<1 ;
$$

- physically represents the variance $D$ of the number of packets in the network, $(n-1)$-fold multiplied in accordance with a given value of the parameter $H$;

- in the operational plan is the coefficient of multiplying the average length of the "exponential" queue $L_{1}$ up to the size of the queue $L_{n}$ of Norros QS $f B / M / 1 / \infty, L_{n}=m \cdot L_{1}$;

- in accordance with Little is true (correct) and for the average waiting time for the packet, $W_{n}=m \cdot W_{1}$;

- scale-invariant $(m=1)$ with respect to the Hurst parameter when loading at the point of the "golden" section $\rho^{*} \approx 0,382$;

- as will be shown below, it is correct for any real values of the Hurst $H$ parameter and the progression $n$ index ;

- it is graphically represented by a family of curves (Figure 1) in the range of the load change of $40 \% \leq \rho \leq 95 \%$ of the server for different values of the Hurst parameter, $H \geq 0,5$.

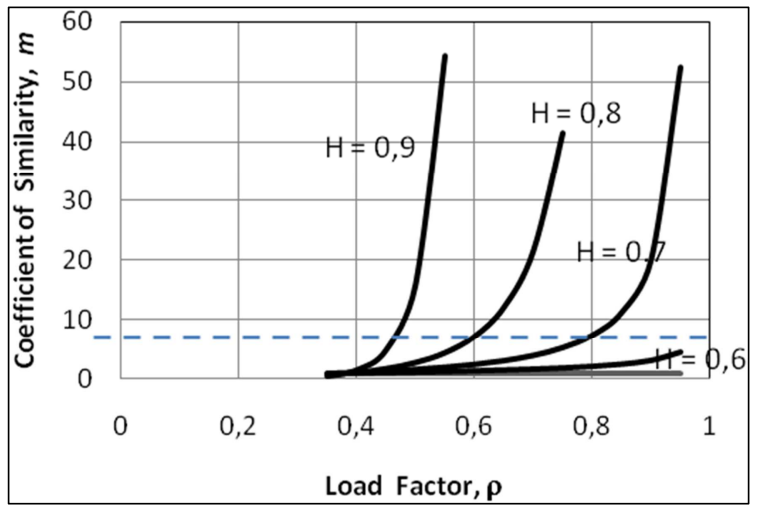

Figure 1. The similarity coefficient, as a function $m(H, \rho)$ of the Hurst parameter and the load.

Analysis of the family of curves (Figure 1) shows that the similarity coefficient $m$ :

- for Poisson traffic by definition, is 1 (see the lower horizontal line);

- for self-similar traffic (see area $H>0.5$ and $\rho \geq 0,4$ ) is always greater than 1 ;

- being a function $m(H, \rho)$, it has a threshold character. Up to some threshold value $\rho^{*}$ this function is relatively constant, but then it increases without limit. If necessary, the threshold value $\rho^{*}$ can be determined from equation (10);

- shows the multiplicative effect of the growth of the queue, due to the non-linear influence of factors (Hurst parameter and load) on packet delay and queue.

Table 2. Coefficient of similarity $m$ depending on load and degree of self-similarity.

\begin{tabular}{llllll}
\hline \multirow{2}{*}{ Server load $\boldsymbol{\rho}$} & \multicolumn{4}{l}{ The value of the similarity coefficient $\boldsymbol{m}$ for the Hurst parameter $\boldsymbol{H}$} & \multirow{2}{*}{ The average length $L_{1}$ of the Poisson queue } \\
\cline { 2 - 4 } & $\mathbf{0 , 5}$ & $\mathbf{0 , 7 5}$ & $\mathbf{0 , 8 3 3 3 \ldots}$ & $\mathbf{0 , 8 7 5}$ & 0,9 \\
0,6 & 1 & 3,75 & 4,1 & 52,7 & 3,2 \\
\hline
\end{tabular}

Table 2 shows that knowing the average length $L_{1}$ of the "exponential" ("Poisson") queue and the similarity coefficient $m$, it is easy to find the average buffer capacity $L_{n}$ under conditions of self-similar traffic. 
Example 2. Suppose given: server load $\rho=0,8$; the value of the Hearst parameter obtained by apreliminary modeling is $H=0.875$; calculated by the formula (10), the similarity factor $m=8000$. To find the buffer capacity $L_{n}$ for selfsimilar traffic, it is sufficient to rescale the "Poisson" solution by multiplying $L_{n}=L_{1} \cdot m=3,2.8000=25600$ (packets).

The presence of pure self-similar traffic in the multi-flow makes it difficult to select a work point (Figure 1). For example, if we limit the maximum allowable value $m$ to tenfold $(m=10)$ by exceeding the "Poisson" length of the queue, then for $H=0.7$ the system can be loaded no more than $\rho^{*} \leq 84 \%$, for $H=0.8$ no more than $63 \%$, for $H=0.9$ the allowable load threshold is lowered to $48 \%$. Exceeding this threshold $\rho^{*}$ will lead to an uncontrolled increase in the length of the queue.

The recurrent delay model (8), (9) is characterized by power law dependence, it is recursive computationally, it is invariant with respect to dimension $n$, it is endowed with the similarity property with the similarity coefficient $m$, but it cannot be recognized as a "full" fractal model, because it is built only for integer index values $n$.

\subsection{The Case of Fractional Indices}

Interpolation of the integer recurrence model (8), (9) in the case of a fractional index $n$ implies the presence in the numerical sequence ${ }_{n}$, in addition to members with integer indices $W_{1}, W_{2}, W_{3}$. as well as delays with a fractional index, for example, $W_{1,9}$ and $W_{4,144}$.

By a fractional geometric progression we mean such a sequence of numbers (members of the progression) in which each successive number is obtained from the previous one by multiplying by the denominator $\tilde{q}$ of the progression, which is the denominator of the integer progression $q$ raised to the power $1 / \tau$,

$$
\tilde{q}=q^{1 / \tau},
$$

where: $1 / \tau$ is the step of crushing, $\tau>1$.

When crushing, the interval between adjacent points of the numerical axis is reduced by a factor of $\tau$. To $n$ integer points, intermediate points $(n-1) \cdot(1-\tau) / \tau$ are added, with a fractional index.

For example, if you set step $1 / \tau=0,001$ to 5 integer points, intermediate points $(5-1)(1-0,001) / 0,001=3996$ will be added, and the denominator of the integer progression, initially equal, say, $q=2$, will decrease to the value $\tilde{q}=$ 1,000693 for a fractional progression. With decreasing pitch $1 / \tau$, the density of the covering of the segment $[0,5 \ldots 1)$ of the numerical axis $\mathrm{H}$ increases, the interpolation error $\rightarrow 0$.

Table 3, obtained for exponential service $(d=1)$, average packet processing time $h=1 \mathrm{~ms}$, server load $\rho=0.6$ and changing incoming self-similar traffic ( $H$-var) reflects the behavior of the recurrence model parameters for integer and fractional index values $n$ from the interval $(1-2,01)$ with a step of crushing $1 / \tau=0,01$.

Table 3. Coefficient $m$ of similarity and average delay ${ }_{n}$, depending on fractional index $n$.

\begin{tabular}{llll}
\hline Hurst parameter $H$ & Index $\boldsymbol{n}$ & Coefficient of similarity & Average access delay $W$ \\
\hline 0,5 & 1 & 1 & 1,5 \\
0,50495 & 1,01 & 1,01330 & 1,52 \\
0,50980 & 1,02 & 1,02678 & 1,54 \\
$\ldots \ldots$ & $\ldots$. & $\ldots \ldots$. & $\ldots$. \\
0,74747 & 1,98 & 3,65216 & 5,48 \\
0,74874 & 1,99 & 3,70076 & 5,55 \\
0,75 & 2 & 3,75 & 5,62 \\
0,75124 & 2,01 & 3,79989 & 5,7 \\
\hline
\end{tabular}

Note: The first line corresponds to a Poisson flow, the delay of which was calculated by the formula $W_{1}=h \cdot \rho /(1-\rho)=1 \cdot 0,6 /(1-0,6)=1,5 \mathrm{~ms}(\mathrm{QS} M / M / 1 / \infty)$. The penultimate line $(H=0.75)$ corresponds to the integer case $(n=2)$. The remaining values of Table. 3 correspond to a fractional "non-Poisson" problem.

The "fractional" calculation algorithm starts with the Hearst parameter $H$ set by the conditions. Next, in sequence, the fractional index $\tilde{n}$, the similarity coefficient $m$, and the average access delay $W_{n}$ are calculated as the $\tilde{n}$-th member of a fractional geometric progression. According to the obtained results, based on the known formulas of Little and Kleinrock, it is easy to define: the average queue length $L=\lambda \cdot W_{n}$, the average time $T=h+W_{n}$ of the packet stay in the server, and the average number of packets in the server $\hat{N}=\lambda \cdot T$.

Example 3. Under the conditions of Table. 3 the "fractional" algorithm takes the form:

1) the value of the Hurst parameter is set, for example, $H$ $=0.74874$;

2) the corresponding value of the index $\tilde{n}$ is calculated taking into account the fragmentation of the interval $\tilde{n}=\frac{\tau}{2 \cdot(1-H)}=\frac{100}{2 \cdot(1-0,74874)} \approx 199 ;$

3) there is a denominator of the integer progression (in fact, the variance)

$$
q=\frac{\rho}{(1-\rho)^{2}}=\frac{0,6}{(1-0,6) \cdot(1-0,6)}=3,75 ;
$$

4) there is a denominator of a fractional progression

$$
\widetilde{q}=q^{1 / \tau}=3,75^{0,01}=1,0133 ;
$$

5) the similarity coefficient is determined $m=\widetilde{q}^{(\tilde{n}-\tau)}=1,0133^{(199-100)} \approx 3,70 ;$ 
6) the average access delay is $W_{n}=m \cdot W_{1}=3,7 \cdot 1,5 \approx 5,55$ ms.

It should be noted that the value $m=q^{n-1}=3,75^{0,5 /(1-H)-1}=3,7$ is the same for both integer and fractional cases, which indicates the invariance of the similarity coefficient.

The choice of the step size $1 / \tau$ of the fragmentation in the delay $W_{n}$ computation problem under consideration is not a critical problem and can in general be excluded from consideration due to the invariance of the similarity coefficient $m$ with respect to the step $1 / \tau$

$$
m=q^{n-1}=q^{\left[\frac{(n-1) \tau}{\tau}\right]}=(\sqrt[\tau]{q})^{(n-1) \tau} .
$$

Indeed, when the interval between integer points is fragmented (the step $1 / \tau$ decreases), the numerator $(n-1) \cdot \tau$ and denominator $\tau$ of the exponent (12) change in different directions and, mutually compensating each other, leave the value of the similarity coefficient $m$ at the same level.

From this it follows that the integral recursive model $(8$, (9) turns out to be correct also for the case of a fractional index $n$, which allows us to understand index $n$ not only as integer values, but also as fractional ones.

In practical terms, finding the "fractal" access delay for the initial data of the previous example for a given value of the Hurst parameter, for example, $H=0.74874$, will be reduced to a preliminary finding of the average "exponential" delay $\left(W_{1}=1.5 \mathrm{~ms}\right)$, variance $(q=3.75)$ and their subsequent multiplication taking into account the exponent $(n-1)$

$$
W_{n}=W_{1} \cdot q^{n-1}=1,5 \cdot 3,75^{1,98997-1} \approx 5,55 \mathrm{~ms},
$$

where $n=0,5 /(1-H)=0.5:(1-0,74874)=1,98997$ is the fractional index.

\section{Approximation of Probability of Loss}

\subsection{Choice of Distribution Law}

To estimate the probability of packet loss, in practice it is customary to use the complementary distribution function (CDF) $\bar{F}(x)$ of the random variable $x$ [8]. A valid choice of the distribution law (distribution function) of the server access delay for the purposes of analytical modeling of server networks is a complex independent task.

It is known that the largest volume of information of the first approximation can be obtained from the modeling results of design situations on exponential models, which are characterized by: compactness, flexibility, reproducibility, simplicity of machining, the need to collect a small amount of initial primary statistics, high accuracy (while observing the accepted exponential assumptions), the possibility of obtaining fairly simple analytical results in an explicit form.

It is generally accepted that the possibilities of oneparameter probabilistic exponential models with a simple
Poisson flow are limited to short-term correlation dependencies and are unsuitable for modeling of heavy traffic $[1,2]$.

Less than can help the heavy-tailed distributions, such as the distribution of Pareto or Weibull. At the pre-project stage of creating telecommunications, sufficient statistical data has not yet been accumulated and a problem of a well-founded task (choice, assignment) of the numerical value of the form parameter arises.

Desirable mathematical properties is to the distribution function of a two-parameter exponential distribution (TpED) $[11,12]$, which unites a wide family of exponential distributions of the form

$$
F_{2 \mathrm{e}}(x)=1-\exp (-x / \alpha T),
$$

where: $T$-scale parameter;

$\alpha_{-}$shape parameter, which for heavy-tailed distributions, $\alpha>1$.

The classical exponential one-parameter distribution (at $\alpha$ =1) [8] turns out to be a TpED special case (14)

$$
F_{\mathrm{e}}(x)=1-\exp (-x / T)
$$

Interpretation of the parameter $\alpha$ is diverse. In the mechanical aspect, the parameter $\alpha$ can be interpreted as the degree of deformation of a simple Poisson process under the influence of an external action (the second Poisson process). In the functional plan $\alpha$ it is, a kind of, the second parameter of scale, which strengthens the influence of the main one $T$. Physically, the parameter $\alpha$ shows how many times the operation of a real system will be slowed down compared to the idealized mode. Depending on the choice of the nature of the random variable, this can be extended to buffer capacity, access delay, and so on.

\subsection{Approximation of the Distribution Function}

To adapt (14) to the conditions of the problem of estimating the probability of packet loss, we perform the following:

- we give to the parameter $\alpha$ a sense of the similarity coefficient $m$;

- suppose, $h \approx 0$, since for heavy traffic the average waiting time $W$ is significantly higher than the average packet processing time $h$;

- we choose the ideal delay $W_{1}$ as the scale parameter.

As a result, the approximation CDF (in the standard of TpED) takes the form

$$
\bar{F}(x) \approx \exp \left(-\frac{x}{m \cdot W_{1}}\right)
$$

Figure 2 shows how "heavier" the tail of the TpEDdistribution with increasing values of the similarity coefficient $m$. In the limit, for $m \rightarrow \infty$, the probability of the packet loss tends to 1 . The dashed line corresponds to the classical oneparameter exponential distribution of the form (15). 
The distribution (16), preserving the exponential structure, acquired heavy-tailed properties. In comparison with (15): the TpED scale parameter (delay) became larger by a factor of $m$, the variance by a factor of $m^{2}$, the probability of packet loss (the probability that the random variable will exceed a standard value $x)$ by $\exp \left[x \cdot(1-1 / m) / W_{1}\right]$ times.
For the initial data of Example 3, using (8), (16) and other mentioned above formulas, Table 4 is calculated, which shows that replacing the self-similar traffic by the Poisson one leads to an increase in the access delay for a few milliseconds, and the probability of packet loss by more than three orders of magnitude.

Table 4. QoS-indicators of compared systems (for the parameter $x=10 \mathrm{~ms}$ ).

\begin{tabular}{lllll}
\hline Incoming traffic & Similarity coefficient $\boldsymbol{m}$ & Ideal Delay, $\boldsymbol{W}_{1}, \mathbf{m s}$, & Real Delay $\boldsymbol{W}$ ms & Loss probability \\
\hline Poisson & 1 & 1,5 & 1.5 & 0,0013 \\
Self-similar & 3,75 & & 5,625 & 0,1690 \\
\hline
\end{tabular}

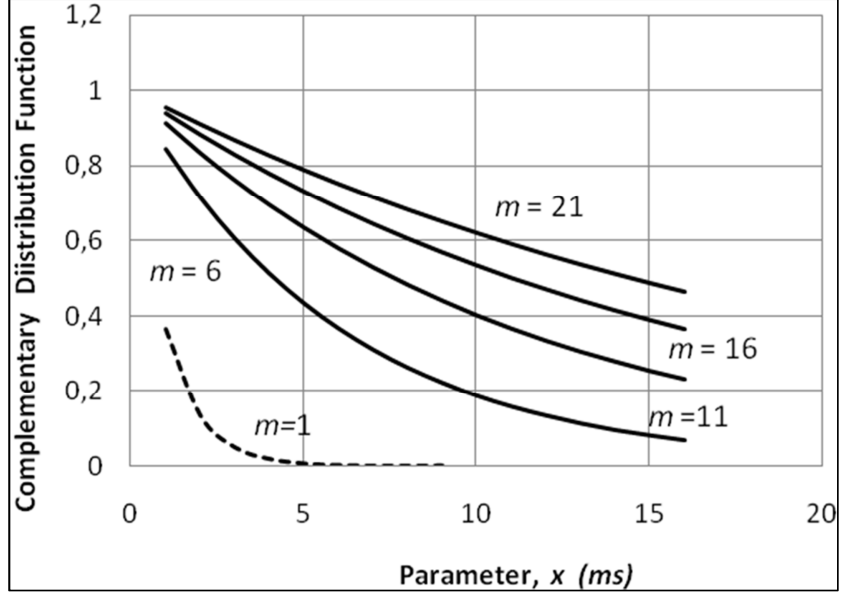

Figure 2. Complementary distribution function for packet loss.

CDF (16) is fundamentally different from CDF (15), although, at first glance, it cannot be said. It can be assumed that after multiplying the parameters $m \cdot W_{1}$, we get a new parameter of the scale of the "lightly-trailing" ("shortly extended") exponent of the form (15), and the self-similarity effect is absent. But this cannot be accepted. Of course, the similarity (in probability of packet loss) of the external forms (15) and (16) is obvious, but not more. In fact, the systems were compared that differ in principle (in $m$ times) speed (reactivity) and performance. All mentioned above is confirmed by Figure 2.

Due to the validity of the identity,

$$
\exp \left(-\frac{x}{m \cdot W_{1}}\right)=\sqrt[m]{\exp \left(x / W_{1}\right)}=\left[\exp \left(-x / W_{1}\right)\right]^{1 / m}
$$

indicating the invariance of the CDF to the location of the index $m$, the following decisive rule can be formulated: "To obtain an estimate from above of the probability of packet loss in a Fractional Brown Motion - type environment, it is necessary to extract the root of the degree equal to the similarity coefficient $m$ from the exponential losses calculated in the conditions of processing Poisson traffic."

For the initial data of Example 3, using formula (17), Table 5 is calculated, which shows that the probability of packet loss is a very critical design factor in comparison with the average access delay.
Table 5. Probability of loss depending on the similarity coefficient $m$

\begin{tabular}{ll}
\hline Similarity Coefficient, $m$ & Probability of Packet Loss \\
\hline 1 & 0,0013 \\
2 & 0,0356 \\
3 & 0,1083 \\
4 & 0,1888 \\
5 & 0,2635 \\
11 & 0,5454 \\
21 & 0,7279 \\
\hline
\end{tabular}

\subsection{Comparison with the Weibull Distribution}

Keeping the properties of the classical exponential distribution, the two-parameter exponential distribution simultaneously acquires the Weibull distribution traits [8]

$$
\bar{F}_{w}(x) \approx \exp \left[-\left(x / W_{1}\right) c\right]
$$

where $c$ is the parameter of the Weibull distribution form.

Comparing the CDFs (16) and (18), it is easy to establish the equivalence condition in the form of a connection equation between the parameter $c$ of the Weibull distribution form and the similarity coefficient $m$ of the recurrence model

$$
c=1-\frac{\ln m}{\ln \left(x / W_{1}\right)}, m<x / W_{1} .
$$

The limiting condition for the applicability of (19) is the similarity coefficient $m$ should not exceed the coefficient $x / W_{1}$ of temporary reservation (the latter can be treated as the ratio of the transaction time-out, the maximum allocated for the transaction of the time interval, to the average time).

It is not difficult to show that the proposed recursive QoS model and the method of rescaling Poisson solutions into fractals based extend to a more general case of nonexponential service $(d \neq 1)$. In the latter case, instead of QS (a single-server QS with a Poisson input flow and exponential service), will appear QS $M / G / 1 / \infty$ (with a Poisson incoming flow and arbitrary service). 


\section{Conclusions}

While the fractal queuing theory is at the embryonic level of development, the authors of this article aimed to maximize the use of the "Poisson" mathematical and software developed over many years for approximate calculation of QoS server metrics with self-similar incoming traffic.

By morphological analysis, became possible to transform the Norros fractional model for the average queue length into a recurrent structure (8), extracting from it Poisson components (average queue length and variance of the number of packets), and also the similarity coefficient (10) of fractal and Poisson stochastic structures.

The similarity coefficient is a new concept that is a scale factor that has the form of a nonlinear function of the server load and the Hurst parameter and allows to establish a correspondence between the parameters of the Poisson and fractal systems.

To assess the probability of packet loss, an additional function of the two-parameter exponential distribution (16) is applied, successfully combining the advantages of exponential and heavy-tailed distributions.

On the basis of the similarity coefficient, a simple method for rescaling Poisson solutions into fractal solutions was constructed. According to this method, to find the fractal delay, it suffices to multiply the exponential delay by the similarity coefficient, and to find the probability of packet loss, extract from the exponential losses the root of the degree equal to the similarity coefficient.

Numerical studies have shown that the resource requirements of self-similar traffic is much higher than the analogous Poisson index, and the probability of packet loss is a more critical design factor than the average access delay.

The proposed method of rescaling "Poisson" solutions into "fractal" solutions requires further development, since covers a far from complete list of QoS parameters of modern infocommunications and models of self-similar traffic, the thesaurus of which, in addition to fractional Brownian motion (FBM), includes fractal Gaussian noise (FGN), chaotic maps (Chaotic Map) models based on the technique of "Dynamic Markov Modeling" (DMM), etc.

\section{References}

[1] O. I. Sheluhin, S. M. Smolskiy, A. V. Osin, "Self-Similar Processes in Telecommunications," Wiley, Chichester, England, 2007, $314 \mathrm{pp}$.

[2] "Self-similar process - Wikipedia“, 2017, Available: https://en.wikipedia.org/wiki/. [Accessed: 8 November, 2017].

[3] I. Norros, "A storage model with self-similar input", Queuing Systems, vol. 16, no. 3, pp. 387-396, 1994.

[4] L. Kleinrok, "Queueing Systems", Volume II: "Computer Applications", John Willey and Sons Inc, 1976, 549 pp.

[5] S. Sh. Kuthbitdinov, "A model for estimating the guaranteed bit rate of multiservice self- similar traffic" / S. Sh. Kutbitdinov, V. V. Lokhmotko, S. R. Rudinskaya // Infocommunications. NetworkTechnologies-Solutions, no. 1, pp. 5-11, 2016 (in russian).

[6] A. I. Kostromitsky, V. S. Volotka, "Approaches to simulating self-similar traffic", 2010. Available:

http://selfsimilar.narod.ru/kostromitsky1.pdf [Accessed: 20 October, 2017] (in Russian).

[7] S. R. Rudinskaya, S. Sh. Kutbitdinov, V. V. Lokhmotko, "Recurrent analogue of the Norros delay model", Modern means of communication, XXII ISTC, Minsk, RB, 2017, pp. 55-57.

[8] C. Forbes, M. Evans, N. Hastings, B. Peacock, "Statistical Distributions", Fourth Edition, John Wiley \& Sons, Inc, 212 p.

[9] M. A. Shneps, "Erlang's first formula as the basis for computing communication networks"/ Collection of scientific papers "Digital communication networks"// Riga, LatvSU named after P. Stuchki, 1989, pp. 21-33 (in Russian).

[10] G. J. Klir, "Architecture of Systems Problem Solving", Plenum Press, New York, 1985, 544 p.

[11] S. Sh. Kutbitdinov, V. V. Lokhmotko, S. R. Rudinskaya, "Estimation of QoS-parameters in the environment of a double stochastic Poisson process // Proceedings of the XVII International Scientific and Technical Conference "Modern Means of Communication", Minsk, 16-18 October 2012, p. 65 (in Russian).

[12] S. Sh. Kutbitdinov, V. V. Lokhmotko, "A model of the queuing system in the environment of a double stochastic Poisson process" // Collected papers of the Fourteenth International Scientific and Practical Conference "Fundamental and Applied Research, Development and Application of High Technologies in Industry and Economics", Volume 1, December 4-5, 2012, SaintPetersburg, Russia, pp. 52-55 (in Russian). 